ISSN 2447-9071

doi https://doi.org/10.36414/rbmc.v6i16.72

Contato para correspondência: Hermínio Maurício da Rocha Sobrinho

E-mail:

herminio.sobrinho@gmail.com

Conflito de interesse: Não

Financiamento: Recursos próprios

Recebido: $27 / 11 / 2020$

Aprovado: 03/12/2020

\section{O uso da toxina botulínica em procedimentos estéticos}

\section{The practical use of botulinum toxin in aesthetics}

Beatriz Nunes Gouveia', Luciana de Lara Pontes Ferreira² ${ }^{2}$ Hermínio Maurício da Rocha Sobrinho $0^{1,2}$

\author{
' Pontifícia Universidade Católica de Goiás - PUC Goiás \\ ${ }^{2}$ Universidade Estadual de Goiás - UEG
}

\section{Resumo}

A toxina botulínica tem como mecanismo de ação a paralisia neuromuscular flácida transitória. Atualmente a toxina botulínica tipo $A$ (TBA) tem sido indicada tanto em procedimentos estéticos quanto terapêuticos. Esteestudoteve porobjetivo descrever as principais aplicações da TBAeseus beneficios em tratamentos estéticos. O presente estudo consisteem uma revisão bibliográfica narrativa. Aaplicação da TBA é um procedimento não cirúrgico, minimamente invasivo, estético terapêutico, temporário, dose-dependente, potente, seguroeeficaz. Éindicada para orejuvenescimento fácil, melhorado sorriso gengival, controle da hiperhidrose, rejuvenescimento escrotal e melhora da aparência de queloides e cicatrizes hipertróficas, com um elevado nível de eficácia e satisfação dos pacientes, cujo efeito pode durar até 6 meses. A técnica de injeção intradérmica ou subdérmica, também conhecida como microbotox ou micro-dosagem, a qual utiliza uma baixa concentração de TBA do que a formulação tradicional para técnica de injeção intramuscular, tem demonstrado uma aparência mais natural no tratamento de rítides/rugas periorbitais, na face eno pescoço. O uso eficaz e seguro da TBA requer um entendimento abrangente da anatomia corporal, experiência prática do profissional, bem como conhecimento e prática sobre a técnica de injeção, localização das aplicações e dosagens adequadas para as áreas a serem tratadas. Dentre os possíveis efeitos adversos do tratamento com a TBA destacam-se o edema, eritema, ptose palpebral, a sensação de pálpebras pesadas, cefaleia, reação inflamatória local e infecção. Portanto, faz-se necessária a capacitação dos profissionais da área da estética para realizarem procedimentos com segurança para minimizaros possíveis efeitos adversos do tratamento.

Palavras-Chave: Toxinas botulínicas tipo A, Estética, Eficácia, Rejuvenescimento.

\begin{abstract}
Botulinum toxin has a transient flaccid neuromuscular paralysis as its mechanism of action. Currently, botulinum toxin type $A$ (BoNT-A) has been indicated for both aesthetic and therapeutic procedures. This study aimed to describe the main applications of BoNT-A and its benefits in aesthetic treatments. The present study consists of a narrative bibliographic review. The application of BoNT-A is a minimally invasive non-surgical procedure, temporary therapeutic aesthetic, dose-dependent, potent, safe and effective. Is indicated for facial rejuvenation, improvement of gingival smile, control of hyperhidrosis, scrotal rejuvenation and reduction of keloids and hypertrophic scars, with a high rate of effectiveness and patient satisfaction, the effect of which can last up to 6 months. The intradermal or subdermal injection technique, also known as microbotox or micro-dosage, which uses a lower concentration of BoNT-A than the traditional formulation for intramuscular injection technique, has shown a more natural appearance in the treatment of periorbital rhytids/wrinkles, on the face and neck. The effective and safe use of BoNT-A requires a comprehensive understanding of body anatomy, practical experience of the professional, as well as knowledge and practice about the injection technique, location of applications and appropriate dosages for the areas to be treated. The main adverse effects of treatment with BoNT-A areedema, erythema, eyelid ptosis, the sensation of heavy eyelids, headache, local inflammatory reaction and infection. Therefore, it is necessary to train professionals in the field of aesthetics to perform procedures safely to minimize the possible adverse effects of treatment.
\end{abstract}




\section{Introdução}

Na última década, os procedimentos estéticos faciais e corporais tornaram-se bastante comuns entre variadas áreas de prestação de serviços à saúde, estética corporal e embelezamento humano. Um número crescente de pacientes tem buscado procedimentos estéticos invasivos mínimos. Um dos procedimentos mais solicitados é o tratamento com toxina botulínica tipo $A(T B A)^{1}$. O tratamento de rejuvenescimento e harmonização facial com TBA é eficaz e produz altas taxas de melhora com início rápido e longa duração de ação. $A$ TBA também tem demonstrado benefícios em tratamento de cicatrizes hipertróficas, rejuvenescimento da região escrotal, rejuvenescimento da região do pescoço, melhora do sorriso gengival, hipertrofia do músculo masseter e sendo usada em microdoses $^{1,2}$.

A toxina botulínica (TB) é uma toxina produzida através da esporulação de uma bactéria gram-positiva e anaeróbica conhecida como Clostridium botulinum ${ }^{3}$, descoberta no ano 1895, ano em que ocorreu um surto de botulismo ${ }^{4}$. O mecanismo de ação da TB consiste em determinar paralisia neuromuscular flácida transitória por meio do processo de denervação química².

A TB pode ser diferenciada em oito sorotipos nomeados como A, B, Cb, C2, D, E, F e G. Comercialmente, estão disponíveis as toxinas tipo $A$ e tipo $B$. Em estética facial, a TBA é a mais utilizada desde que foi aprovada pelo Food and Drug Administration (FDA), em 20025.

ATB é uma neurotoxina utilizada para tratamentos de diversos campos, como: odontológicos, oftalmológicos e neurológicos, porém o que tem estado em alta é o seu uso em tratamentos estéticos. É um procedimento não cirúrgico minimamente invasivo, estético terapêutico temporário, dose-dependente, potente e eficaz em procedimentos estéticos, que vão de rejuvenescimento fácil, até diminuição de queloides e cicatrizes hipertróficas, com uma elevada taxa de eficácia e satisfação das pacientes, cujo seu efeito dura até 6 meses $^{6}$.

Nos Estados Unidos e no Brasil, a TB é um dos procedimentos estéticos mais utilizados para o rejuvenescimento facial ${ }^{6}$. A toxina injetada em pontos específicos da musculatura facial interage com o músculo paralisando-o por um período de 3 a 6 meses. Por ter uma durabilidade esse tratamento requer doses periódicas para que ocorra o prolongamento do seu efeito ${ }^{6}$.

A TBA atua diretamente na paralização do músculo, sendo bastante utilizada no tratamento de rugas existentes, evitandose também o aparecimento de novas rugas faciais, contribuindo para o rejuvenescimento facial5. Além da aplicação do botox em rugas, a TBA pode também ser usada para correção do sorriso gengival, prevenir e tratar queloides e cicatrizes hipertróficas, para tratar hiperidrose, para promover o rejuvenescimento escrotal e para utilização do microbotox. Recentemente o uso da TB tem sido associado com preenchedores dérmicos, tendo revelado potencial para resultados estéticos cada vez melhores, novas utilizações e maior satisfação dos pacientes².

Esta revisão bibliográfica objetivou descrever as principais aplicações da TBA em procedimentos estéticos ressaltando os seus benefícios.

\section{Métodos}

Este trabalho constitui uma revisão bibliográfica narrativa. A pesquisa dos estudos relacionados ao tema foi realizada nas bases de dados eletrônicas National Center for Biotechnology linformation (PubMed/Medline), SciELO, Portal de Periódicos Capes e Google Acadêmico. Foram selecionados trabalhos nos idiomas português e inglês, publicados no período do ano de 2001 ao ano de 2020, que apresentaram conteúdos relacionadas aos objetivos desta pesquisa e que estavam disponíveis na íntegra para leitura. Para a busca dos estudos nas bases de dados foram utilizados as seguintes palavras-chave ou Descritores em Ciências da Saúde (DeCS), em português e inglês com múltiplas combinações: Botulinum Toxins Type A, esthetics, microbotox, rejuvenation. Foram excluídos do estudo os trabalhos publicados fora do período temporal mencionado, os duplicados, os que não apresentavam texto completo disponível nas bases de dados e aqueles não condizentes com os objetivos propostos. Um total de 81 artigos, relacionados ao tema deste estudo, foram encontrados nas bases de dados eletrônicas utilizadas. Deste total, foram selecionados 31 estudos por atenderem aos critérios de inclusão estabelecidos.

\section{Resultados e Discussão}

\section{Envelhecimento Cutâneo}

O envelhecimento cutâneo é um processo que afeta não só a aparência, mas também a função da pele, isso ocorre porque há alteração do material genético e ocorre a diminuição da proliferação celular, que ocasiona a perda de elasticidade, diminuição da replicação dos tecidos e do metabolismo. Pesquisadores apontam o desequilíbrio do mecanismo de defesa antioxidante do organismo como o principal causador do envelhecimento cutâneo ${ }^{7}$. Alguns outros fatores também conhecidos são: idade, hábitos alimentares, sedentarismo, stress, tabagismo, alcoolismo, excesso de sol sem o protetor solar e a poluição. As contrações musculares, apesar de serem as responsáveis pelas expressões do rosto, também são um dos grandes causadores de rugas, conhecidas como vincos, as mesmas podem ser classificadas de duas maneiras: estáticas ou dinâmicos. As estáticas são visíveis na pele mesmo quando não 
há contração da musculatura, são originados pelo excesso da contração muscular, já os vincos dinâmicos aparecem apenas quando há uma contração muscular ${ }^{8}$.

A flacidez tegumentar é um problema que vem com o envelhecimento, ocorre devido à perda de elasticidade e do tônus do tecido. A flacidez ocorre não só decorrente do envelhecimento, mas também por perda de peso, hábitos alimentares, exposição excessiva ao sol e etc. A flacidez tem como fonte a alteração e diminuição das estruturas profundas, mas a consequência ocorre na superfície. Na derme, as fibras colágenas ficam mais espessas e as fibras elásticas ficam sem a sua elasticidade em consequência a diminuição do número de fibroblastos. Na hipoderme, ocorre a diminuição de gordura. Ocorre também diminuição do trofismo e tônus muscular e em fase mais tardia, a massa muscular esquelética começa a ser perdida e substituída por gordura, como exemplo na região submentoniana (pescoço). A frouxidão tecidual provoca a flacidez facial, fazendo com que a pele perca a sua firmeza9.

\section{Aspectos Gerais da Toxina Botulínica (TB)}

ATB é uma neurotoxina produzida pela bactéria Clostridium botulinum (bactéria Gram-positiva da família Bacillaceae, anaeróbica) causadora de uma grave doença, nomeada botulismo, a mesma causa a paralisia dos músculos da face, dos membros e respiratórios, podendo levar ao óbito. A TB atua causando a paralisia neuromuscular flácida transitória² .

A TB causa a inibição nos terminais nervosos motores da liberação exocitótica da acetilcolina causando a diminuição da contração do músculo ${ }^{3}$. A toxina não se liga às fibras nervosas dos troncos nervosos ou da região pós-sináptica. A TB liga-se ao terminal da placa motora. Há evidências de que a cadeia pesada seja a responsável por esta ligação. A ligação acontece no nível dos receptores específicos existentes na membrana da terminação nervosa. A cadeia pesada é neurotrópica, seletiva para as terminações nervosas colinérgicas. A TB é internalizada por endocitose para o endossoma e daí para o citossoma através de um processo onde parece estar envolvido com um sensor de $\mathrm{pH}$ (5,5 ou menos) que ajuda na mudança da configuração da molécula ${ }^{3,10,11}$.

A TB age seletivamente no terminal nervoso periférico colinérgico, inibindo a liberação de acetilcolina. Entretanto, a TB não ultrapassa a barreira cerebral e não inibe a liberação de acetilcolina ou de qualquer outro neurotransmissor no cérebro. A sequência da ação da TB inclui: difusão, neurotropismo, ligação, internalização e toxicidade intracelular que é exercida pela alta afinidade da toxina com os receptores específicos da parede intracelular do terminal pré-sináptico ${ }^{3,10,11}$.

Após a internalização da TB a cadeia leve da molécula é liberada no citoplasma da terminação nervosa. Uma vez no citoplasma da célula, a cadeia leve faz a quebra das proteínas de fusão, impedindo assim a liberação da acetilcolina para a fenda sináptica. Esse processo produz uma denervação química funcional, reduzindo a contração muscular de forma seletiva. A propagação do potencial de ação, a despolarização do nervo terminal os canais de $\mathrm{Na}$, K, e Ca não são afetados pela toxina ${ }^{3,10,11}$.

A duração do efeito é temporária, devido a formação de novos receptores de acetilcolina, quanto mais contatos sinápticos o axônio terminal forma vai há um reestabelecimento da transmissão neuromuscular causando a volta gradual da contração muscular com efeitos colaterais mínimos. Devido a isso, o tratamento com a TB, é um tratamento temporário, dose-dependente e reversível, entretanto, é um procedimento que exige um profissional devidamente habilitado e capacitado para seguir os protocolos de aplicação corretamente no prazo de 6 em 6 meses para que não ocorra o risco de uma resposta secundaria a toxina ${ }^{2,12}$.

\section{Uso da Toxina Botulínica tipo A (TBA) em Procedimentos Estéticos}

A TBA é uma neurotoxina utilizada para tratamentos de diversos campos, tais como: odontológicos, oftalmológicos e neurológicos, porém o que está em evidência é o seu uso em tratamentos estéticos. A aplicação da TBA é um procedimento não cirúrgico minimamente invasivo, estético terapêutico temporário, dose-dependente, potente e eficaz, sendo indicada para o rejuvenescimento fácil, melhora do sorriso gengival, controle da hiperhidrose, rejuvenescimento escrotal e diminuição de queloides e cicatrizes hipertróficas, com uma elevada taxa de eficácia e satis fação das pacientes, cujo seu efeito dura até 6 meses $^{6}$. As marcas de Botox aprovadas pela Agência Nacional deVigilância Sanitária (ANVISA) são: Botox ${ }^{\oplus}$, Xeomin ${ }^{\oplus}$, Prosigne ${ }^{\oplus}$, Dysport ${ }^{\oplus}$, Botulift ${ }^{\oplus}$. A administração da TBA se dá por via intramuscular/intradérmica conforme protocolo técnico da área a ser tratada ${ }^{13}$. A duração do efeito ocorre de 6 semanas até 6 meses, atingindo os melhores resultados entre 2 a 3 meses $^{6}$.

Os principais músculos que são submetidos a aplicação da TBA são os seguintes: músculo frontal, corrugador do supercílio, orbicular do olho, prócero, músculo nasal, levantador do lábio superior e da asa do nariz, levantador do lábio, zigomático menor, zigomático maior, levantador do ângulo da boca, bucinador, risório, orbicular dos lábios, depressor do ângulo da boca, depressor do lábio inferior e músculo mentoniano ${ }^{14}$.

A aplicação da TBA apresenta riscos, mas os efeitos adversos são geralmente leves e passageiros, tendo a duração de alguns dias após a aplicação e podem ser evitados quando 
obedecidos os protocolos técnicos, respeitando as normas e as indicações, realizados por um profissional experiente. Os efeitos adversos podem ocorrer no local da aplicação ou até mesmo em locais distantes da aplicação, estes incluem: hematomas, dor, parestesia, sensibilidade, inflamação, hipoestesia, edema, infecção localizada, eritema, hemorragia ou ardor associados a injeção, tanto no local quanto no músculo adjacente, fraqueza no músculo local e também adjacente ${ }^{5}$. As principais aplicações da TBA em procedimentos estéticos estão apresentadas na figura 1, a seguir.

Figura 1. Principais aplicações da Toxina Botulínica tipo A (TBA) em procedimentos estéticos.

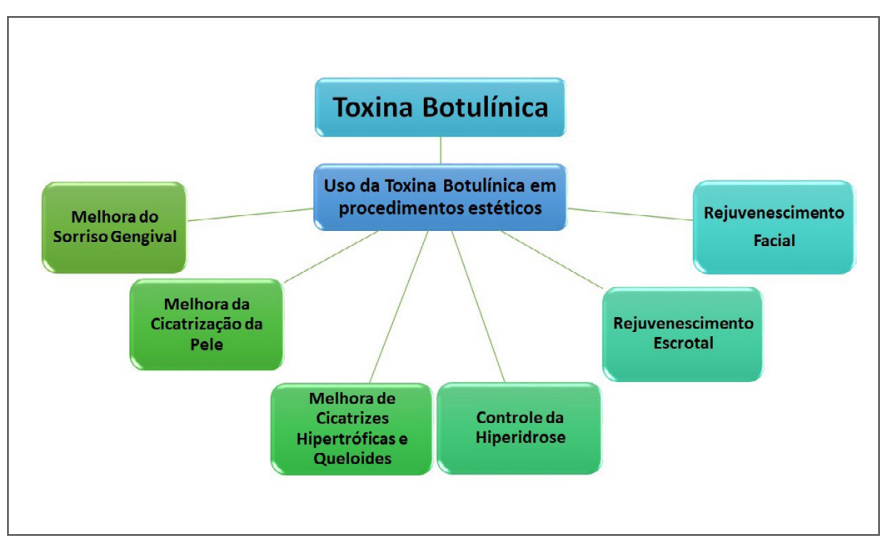

\section{Melhora do Sorriso Gengival}

O sorriso é um dos maiores atrativos do rosto, sendo utilizado para expressar emoções e até para demonstrar sensualidade. Anatomicamente, o sorriso é a contração de um conjunto de músculos, localizados no terço médio e inferior do rosto, que causam a exposição dos dentes e de 1 a $2 \mathrm{~mm}$ da gengiva. Um sorriso é considerado um sorriso gengival quando mais do que $2 \mathrm{~mm}$ de gengiva aparecem durante $\mathrm{o}$ ato de sorriso, causando uma desarmonia no sorriso, que traz incômodo aos pacientes ${ }^{15-17}$.

Para fazer o diagnóstico do sorriso gengival é necessário que ocorra uma classificação do nível gengival, para isso é necessário levar em conta variáveis, como: idade, gênero e saúde periodontal dos pacientes. Em seguida, é preciso estabelecer a etiologia do sorriso gengival, que normalmente é uma junção de fatores, mas alguns dos fatores são; contração excessiva dos músculos do terço médio, crescimento vertical excessivo do maxilar, comprimento ou largura desproporcionais da coroa dentaria dos dentes anteriores e lábio superior com comprimento inferior ao proporcional ${ }^{15-17}$.

Cirurgia odontológica pode resolver o sorriso gengival, porém, uma opção minimamente invasiva que também pode ajudar, ou até mesmo, substituir a cirurgia é a aplicação da TBA para tratar o sorriso gengival, uma vez que a TBA impede a contração muscular e relaxa a musculatura dos lábios ${ }^{15,16,18}$.

A aplicação da TBA para a harmonização do sorriso gengival pode ser feita por profissionais dentistas especializados em estética, uma vez que é necessário que seja feita a análise dentaria do paciente, assim como o exame do lábio superior. Os músculos envolvidos no sorriso são: elevador do lábio superior, zigomático maior e o menor e o músculo da asa do nariz. Porém, é necessário ressaltar que cada músculo possui uma determinada função no sorriso, então, cada tipo de sorriso gengival envolve um determinado músculo ${ }^{18}$.

O procedimento de aplicação da TBA para o sorriso gengival é um recurso rápido e seguro se comparado ao procedimento cirúrgico, gerando um resultado harmônico quando a aplicação é feita corretamente no músculo alvo. Para que a aplicação seja bem sucedida é preciso seguir um protocolo, começando com a avaliação adequada do paciente, levando em conta os aspectos mencionados anteriormente $\mathrm{Em}$ seguida é feita a marcação de onde ocorrerá a aplicação, a marcação deve ser feita enquanto o profissional orienta o paciente a dar sorrisos forçados para melhor avaliar onde necessita de aplicação. A dosagem usada pode ser de $2 \mathrm{U}$ a $3 \mathrm{U}$ por ponto para pessoas com 3 a $5 \mathrm{~mm}$ de gengiva exposta. A aplicação é feita com uma seringa estéril de $1 \mathrm{ml}$ com resíduo zero acompanhada de uma agulha, também estéril, de $6 \mathrm{~mm} / 32 \mathrm{G}$ lembrando que deve ser feita a aplicação igualmente em ambos os lados, caso use um anestésico tópico, sua aplicação deve ser feita de 15 a 20 minutos antes da aplicação ${ }^{18}$.

\section{Uso Da TBA para Melhora da Cicatrização da Pele}

A TBA pode ser usada para melhorar a cicatrização cutânea e evitar queloides e cicatrizes hipertróficas, pois a TBA reduz a tensão das bordas da ferida durante a cicatrização dando um melhor aspecto a cicatriz ${ }^{19,20}$. Esta melhora se dá pela ligação inflexível da neurotoxina com à junção neuromuscular, que como consequência causa a inibição da liberação pré-sináptica de acetilcolina19. Sendo assim sua capacidade de suprimir a contração muscular pode ser usada para reduzir a tensão nas bordas das feridas ou incisões, causando também a diminuição da proliferação de fibroblastos, inibindo a síntese de colágeno tipo I pelos fibroblastos, principal componente da matriz extracelular ${ }^{21}$.

ATBA é colocada em frascos em uma proporção de $0,6 \mathrm{ml}$ de solução salina junto com 15 unidades de TBA, ou seja, $4 \mathrm{ml}$ de solução salina por 100 unidades de TB, em seguida as injeções com o TBA são feitas na musculatura subjacentes a ferida cujo visando uma melhor cicatrização, a aplicação é feita em um diâ- 
metro de 1 a $3 \mathrm{~cm}$ ao redor das bordas da ferida, com o intuito de que os músculos sejam paralisados durante a cicatrização, diminuindo a tensão na ferida reduzindo as chances ou até evitando a formação de uma cicatriz grosseira ${ }^{20,22}$.

\section{Uso da TBA em Queloides e Cicatrizes Hipertróficas}

As queloides e as cicatrizes hipertróficas causam uma aparência inestética em regiões corporais afetadas nos pacientes ${ }^{2}$. Ambos os tipos de lesões são causados por distúrbios da cicatrização decorrentes de respostas inflamatórias excessivas durante a cicatrização da lesão. Neste processo ocorre a perda do controle entre síntese e degradação do colágeno, a patogênese das queloides e cicatrizes hipertróficas não foi completamente desvendada, porém, fatores como origem étnica, localização, fatores neurais, atrasos na epitelização, inflamação prolongada, citocinas, ação de músculos pericicatriciais, forças de tensão da pele adjacente e também infecção podem ser relacionados com a formação destas lesões $2,19,23,24$.

Visualmente, a diferença entre as duas se dá porque as queloides ultrapassarem os limites das incisões da ferida, já as cicatrizes hipertróficas geralmente são elevadas, mas raramente se elevam a mais de $4 \mathrm{~mm}$ acima da pele, tem a cor avermelhada ou rosada, são duras, pruriginosas e não se estendem além das margens gerais da ferida ${ }^{19}$. Histologicamente, elas não possuem diferenças, na microscopia eletrônica, é possível observar substância amorfa ao redor dos fibroblastos nas queloides, além de maior atividade metabólica e anticorpos antinucleares ${ }^{23}$.

O uso da TBA para a melhoria das cicatrizes se dá pelo relaxamento dos músculos ao redor da ferida, diminuindo assim a tensão dos músculos através da aplicação de $10 \mathrm{U}$ de TBA por $0,2 \mathrm{ml}$ de salina em ambos os lados da cicatriz a $5 \mathrm{~mm}$ para cada $1 \mathrm{~cm}$ de cicatriz ${ }^{2,24}$.

\section{Uso da TBA no Controle da Hiperidrose}

A hiperidrose é uma patologia que atinge $3 \%$ da população adulta $^{25}$, onde ocorre a hiperprodução de suor decorrente das glândulas sudoríparas dos pacientes serem hiperfuncionais, pode ser associada a fatores hereditários, ocupacionais, emocionais e até sociais. A hiperidrose pode atingir regiões especificas do corpo, ou ser localizada, pode ocorrer no couro cabeludo, face, axilas, palmas das mãos, virilhas e plantas dos pés, sendo mais comum nas axilas. Pode ser classificada em primária (perceptível na infância ou adolescência, não acontece em repouso ou durante o sono, geralmente atinge as axilas, mãos e pés) e em secundária (surge na fase adulta, ocorre mesmo em repouso e durante o sono, pode ser causada por comorbidades ou por efeito colateral de medicações, pode ocorrer em todas as áreas do corpo). Há duas opções de tratamento que levam em conta o grau da hiperidrose, pode ser usado medicamentos para tratar de maneira não invasiva, como por exemplo, usando antiperspirantes, ou tratando com cirurgia de maneira invasiva. A TBA também vem sendo usada para realizar esse tratamento e tem se mostrado eficaz ${ }^{25-27}$.

A TBA tem sido utilizada para o tratamento da hiperidrose e tem sido bastante eficaz devido sua ação como agente neuroparalítico, causando paralisia muscular por bloquear a transmissão colinérgica dos terminais nervosos pré-sinápticos, que inibem a exocitose de acetilcolina na fenda pré-sináptica ${ }^{26}$.

As glândulas sudoríparas são inervadas por fibras simpáticas pós-ganglionares, a TBA se aglutina nas terminações nervosas dessas fibras sendo internalizada por endocitose e em seguida liberada no citoplasma axonal. As glândulas sudoríparas não recebem os estímulos para secreção, pois a toxina impede a liberação de neurotransmissores seccionando a proteína SNAP-25 (proteína de membrana celular), usada para liberar a acetilcolina na fenda pré-sináptica, reduzindo a secreção de suor ${ }^{27}$.

\section{Uso da TBA no Rejuvenescimento Escrotal}

Os escrotos são uma bolsa de músculo-cutânea que são separados em dois lóbulos onde estão contidos os testículos. São revestidos pela Túnica de Dartos (um músculo cutâneo formado por fibras musculares lisas e fáscia) e a Cútis, pele que reveste o escroto, a qual é fina e enrugada, apresenta pregas transversais, na linha mediana encontramos a rafe do escroto ${ }^{2,28}$.

Com o envelhecimento o escroto acaba por se tornar flácido e rugoso (pendurado e enrugado), o que para muitos homens pode causar não só um desconforto estético, mas também fisiológico, uma vez que o escroto flácido continuamente fricciona entre as coxas causando um desconforto, além de que podem sentir dor durante a relação sexual, para se exercitar e na de pratica esportes ${ }^{28}$.

Para a melhora do aspecto morfológico escrotal tem a opção cirúrgica em que ocorrerá a remoção do excesso de pele, porém, para a resolução do escroto rugoso, a alternativa de tratamento é a aplicação da TBA. Sabe-se que as rugas são acentuadas pelas contrações do músculo dartos, que ocorre como uma resposta a baixas temperaturas ou a relação sexual. Como o músculo dartos é um músculo liso e a atuação da TBA se dá pela paralização de músculos lisos, a aplicação da TBA é capaz de intervir diretamente na função do músculo amenizando as rugas e dando um melhor aspecto ao escroto alisando a pele da superfícieie,28.

\section{Microbotox}

O procedimento de Microbotox foi desenvolvido no ano de 2000, primeiramente a técnica foi nomeada como Mesobotox 
devido a sua semelhança com a Mesoterapia, porém, com o tempo, ocorreu a percepção de que o termo não era adequado, pois não transmitia a extensão ou profundidade das injeções feitas no procedimento. Posteriormente o termo Microbotox foi escolhido para refletir a quantidade de TBA em cada microgotícula aplicada nas áreas-alvo ${ }^{29}$.

Um dos procedimentos mais comuns da aplicação da TBA na prática estética é a técnica de injeção intradérmica, que tem demonstrado fornecer benefícios em termos de melhora da textura da pele e o rejuvenescimento facial ${ }^{30}$. A técnica de injeção intradérmica ou subdérmica, também conhecida como "micro-dosagem", a qual envolve o uso de uma concentração mais baixa de TBA do que a formulação tradicional para técnica de injeção intramuscular, que é injetado em várias alíquotas minúsculas sobre as áreas corporais tratadas ${ }^{29}$.

O microbotox vem sendo bastante aplicado em rítides periórbitais localizadas no terço inferior do músculo orbicular dos olhos. Entretanto, podem ser aplicados na face e no pescoço ${ }^{29,31}$. As aplicações são realizadas por meio de múltiplas pequenas bolhas de TBA na pele ou nos músculos faciais em distâncias de 0,8 a $1,0 \mathrm{~cm}^{29}$.

A técnica de microdoses é bastante utilizada na testa e sob os olhos, uma vez que não tem como intenção causar a paralisia do músculo e sim a suavização de linhas finas e rugas, além de diminuir os efeitos de puxar e amarrar os músculos. As microdoses enfraquecem as fibras musculares superficiais da região inferior da pele, evitando que a toxina interaja com os músculos mais profundos. Como consequência, o microbotox dá uma aparência mais natural, quando comparado com o botox em que a aplicação na testa fica mais rígida e imóvel ou pálpebras inferiores inanimadas ${ }^{29}$.

A TBA precisa ser diluída para seu uso como microdoses, sendo feita a diluição de um frasco de $100 \mathrm{U}$ de TBA em $2 \mathrm{ml}$ de soro fisiológico a $0,9 \%$, em seguida, dessa solução é retirada duas unidades de $0,04 \mathrm{ml}$ acrescentando $0,40 \mathrm{ml}$ de soro fisiológico em seringa BD Ultra Fine II curta de $1 \mathrm{ml}$ e agulha de $8 \mathrm{~mm}$, o que completa um volume total de $0,48 \mathrm{~m}^{31}$.

A aplicação é feita após a avaliação e classificação das rugas periorbitais, as quais são classificadas em Tipo I, Tipo II, Tipo III, de acordo com a localização corporal, conforme apresentado a tabela 1, e ainda subclassificadas em tipos $A$ e $B$, sendo as do tipo $B$ dividas em $B 1, B 2$ e $B 3$, conforme a figura $2^{31}$.
Tabela 1. Classificação das rugas em três tipos de acordo com a localização anatômica.

\begin{tabular}{|c|c|c|}
\hline Tipo I & Tipo II & Tipo III \\
\hline $\begin{array}{c}\text { Rugas laterais ao canto } \\
\text { externo do olho, esten- } \\
\text { dendo-se da sobrancelha } \\
\text { até o arco zigomático. } \\
\text { to externo do olho, } \\
\text { estendendo-se da linha } \\
\text { do canto externo do olho } \\
\text { até o arco zigomático } \\
\text { (ausência de rugas na re- } \\
\text { gião lateral superior). }\end{array}$ & $\begin{array}{c}\text { Presença de rugas exclu- } \\
\text { sivamente na linha do } \\
\text { canto externo. }\end{array}$ \\
\hline
\end{tabular}

Fonte: Adaptado de Oliveira; Rossi; Moreira (2016) ${ }^{31}$.

Figura 2. Subclassificação das rugas Periorbitais. Adaptado de Oliveira; Rossi; Moreira (2016)31.

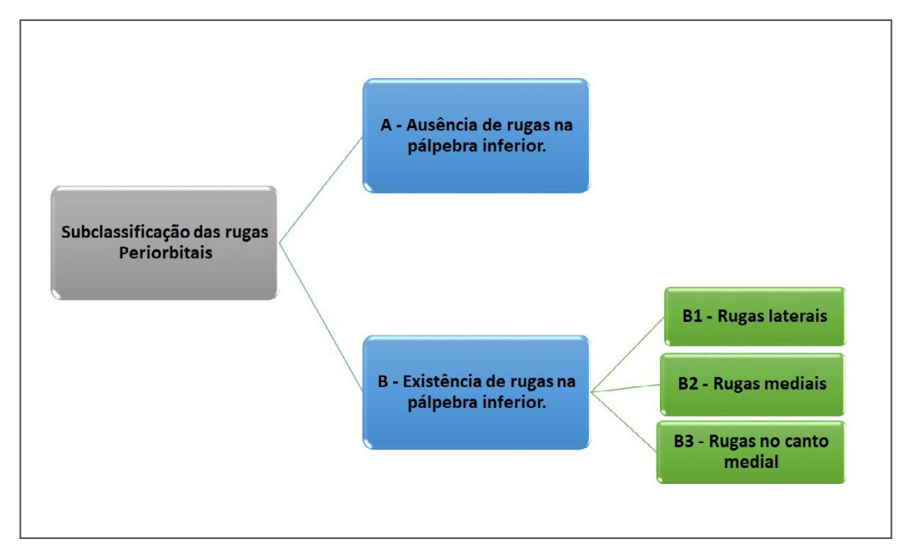

O número de microdoses aplicada varia para cada paciente, pois depende da força muscular da região e da formação de rítides dinâmicas, em alguns casos é necessário a realização de mais aplicações no retorno do paciente 15 dias depois da primeira aplicação, porém os resultados mostram a eficácia e a segurança da aplicação das microdoses nas rítides periorbitais localizadas no terço inferior do músculo orbicular dos olhos ${ }^{31}$.

\section{Conclusão}

Os tratamentos com toxina botulínica tipo A (TBA) tem sido considerados eficazes, seguros, apresentando resultados satisfatórios. As aplicações da TBA para rejuvenescimento facial têm sido bem estabelecidas em todo o mundo, especialmente, para o tratamento de rugas. Estudos estão identificando e demonstrando o uso da TBA para outros fins, tanto no campo estético quanto no terapêutico, tais como para o tratamento da hiperidrose, melhora de cicatrizes cutâneas e de patologias dermatológicas.

O uso da TBA em procedimentos estéticos faciais, apresentam altas taxas de melhora com rápido início de ação e longa 
duração de ação (mais de 4 meses para a maioria dos pacientes) quando comparado ao placebo.

As microdoses da TBA (microbotox) se mostraram mais eficazes para o tratamento de rugas na testa e nos olhos, dando um aspecto mais natural ao paciente, quando comparado ao botox. A TBA tem sido muito usada por pacientes para diversos tratamentos e está sendo muito bem aceita e quando usada da forma correta, satisfazendo os pacientes.

O uso eficaz e seguro da TBA requer um entendimento abrangente da anatomia corporal, experiência prática do profissional, bem como conhecimento e prática sobre a técnica de injeção, localização das aplicações e dosagens adequadas para as áreas a serem tratadas.

Estudos demonstraram que as diferentes indicações estéticas da aplicação da TBA estão associadas a níveis consistentes e significativamente elevados de satisfação dos pacientes, o que se correlaciona com a eficácia do tratamento e a incidência de efeitos adversos que é, relativamente, baixa.

\section{Referências}

1. Schlessinger J, Gilbert E, Cohen JL, Kaufman J. New uses of abobotulinumtoxina in aesthetics. Int Aesthetic Surg J. 2017;37:45-58.

2. Vasconcellos RC, Sotero P, Lage R. Atualizações do uso cosmiátrico e terapêutico da toxina botulínica. Int Surg Cosmet Dermatology. 2019;10(3):97-104.

3. Sposito MMM. Toxina Botulínica do Tipo A: mecanismo de ação. Rev Acta Fisiátrica. 2009;16:25-37.

4. Fujita RLR, Hurtado CCN. Aspectos relevantes do uso da toxina botulínica no tratamento estético e seus diversos mecanismos de ação. Int Saber Científico. 2019;8(1):120.

5. Santos CS, Mattos RM, Fulco TO. Toxina botulínica tipo A e suas complicações na estética facial. Rev Episteme Transversalis. 2015;6(2236-2649):73-84.

6. Bratz PDE, Mallet EKV. Toxina Botulínica tipo A: abordagens em saúde. Rev Saúde \& Ciência em Ação 2016;3(2447-7079):58-70.

7. Lima L, Pinto DO, Priscila D, Mejia M. Envelhecimento cutâneo facial: radiofreqüência, carboxiterapia, correntes de média frequência, como recursos eletroterapêuticos em fisioterapia dermato - funcional na reabilitação da pele - revisão de literatura . Int Fac Ávila. 2009. [acesso em 2020 set 22]. Disponível em: https:// portalbiocursos.com. br/ohs/data/docs/14/13_Envelhecimento_CutYneo_Facial_RadiofreqYYncia_carboxiterapia_correntes_de_mYdia_frequYncia.pdf.

8. Paulo EV , Oliveira RCG. Avaliação e sugestão de protocolo estético para aplicação de toxina botulínica do tipo
A em pacientes adultos. Rev Uningá. 2018;55:158-67..

9. Silva SA, Pinto LP, Bacelar IA. O uso da radiofrequência no rejuvenescimento facial - revisão de literatura. Rev Saúde em Foco. 2018;569-80.

10. Mattos A. Toxina botulínica tipo A recomendações, contraindicações e mais. Int PUBMED. 2018. [Acesso em 2020 out 23]. Disponível em: https://pebmed.com. br/toxina-botulinica-tipo-a-recomendacoes-contraindicacoes-e-mais/.

11. Satriyasa BK. Botulinum toxin (Botox) a for reducing the appearance of facial wrinkles: A literature review of clinical use and pharmacological aspect. Int Clin Cosmet Investig Dermatol. 2019;12:223-8.

12. Martins RR, Silveira AMM, Raulino Neto JS, Martins JCG, Pessoa CV. Toxina Botulínica tipo A no tratamento de rugas. Int Most Científica da Farmácia Cent Univ Católica Quixadá - Unicatólica. 2016;3(2358-9124).

13. Allergan Produtos Farmacêuticos. Botox: bula para o profissional de saúde. 2019. [Acesso em 2020 out 28]. Disponível em: https://allergan-web-cdn-prod. azureedge.net/allerganbrazil/allerganbrazil/media/ allergan-brazil/botox_bula_profissional.pdf.

14. Oliveira G. Toxina Botulínica e suas complicações: uma revisão de literatura. Int Repositório institucional. 2019:1-41. [Acesso em 2020 mai 27]. Disponível em: https://repositorio.ufsc.br/handle/123456789/201604.

15. Jaspers GWC, Pijpe J, Jansma J. The use of botulinum toxin type A in cosmetic facial procedures. Int J Oral Maxillofac Surg. 2010;40(2):127-33.

16. Pacotto RC, Moreira M. Integração da odontologia com a medicina estética: correção do sorriso gengival. Int RGO-Rev Gaúcha Odontol. 2005;53(1981-8637):171-8.

17. Kuhn-Dall'Magrol A, Calzall SC, Lauxen J, Santos R, Valcanaia TDC, Dall'Magro E. Tratamento do sorriso gengival com toxina botulínica tipo A: relato de caso. Int Rev da Fac Odontol - UPF. 2015;20(1):81-7.

18. Silva Neto JMA, Batista ARC, Barros IRV, Araujo YBM, Duarte IKF, Tenório Neto JF. Protocolos de Aplicação de Toxina para Sorriso Gengival: uma revisão de literatura. Rev Eletrônica Acervo Saúde. 2019;(28):e1079.

19. Kasyanju Carrero LM, Ma WW, Liu HF, Yin XF, Zhou BR. Botulinum toxin type $A$ for the treatment and prevention of hypertrophic scars and keloids: updated review. Int J Cosmetic Dermatology. 2019;18(1):10-5.

20. Chang CS, Wallace CG, Hsiao YC, Chang CJ, Chen PK. Botulinum toxin to improve results in cleft lip repair: $A$ double-blinded, randomized, vehicle-controlled clinical trial. Int PLoS One. 2014;9(12):e115690. 
21. Cardoso AS, Teixeira DA, Oliveira BV, Carneiro PP, Junqueira RF. Aplicação de toxina botulínica na cicatrização por segunda intenção. Int Surg Cosmet Dermatol. 2016;8(2):163-6.

22. Gassner HG, Brissett AE, Otley CC, Boahene DK, Boggust $A J$, Weaver $A L$, et al. Botulinum toxin to improve facial wound healing: A prospective, blinded, placebocontrolled study. Int Mayo Clin Proc. 2006;81 (8):1023-8.

23. Campos ACL, Borges-Branco A, Groth AK. Cicatrização de Feridas. Int ABCD, Arq. Bras. Cir. Dig. 2007;20(1):51-8.

24. Rocha D. A toxina botulínica e sua aplicação em cicatrizes faciais. Int PUBMED. 2018. [Acesso em 2020 nov 16]. Disponível em: https://pebmed.com.br/a-toxinabotulinica-e-sua-aplicacao-em-cicatrizes-faciais/.

25. Bernhard MK, Krause M, Syrbe S. Sweaty feet in adolescents - Early use of botulinum type A toxin in juvenile plantar hyperhidrosis. Int Pediatric Dermatology. 2018;35(6):784-786.

26. Hagemann D, Sinigaglia G. Hiperidrose e o uso da toxina botulínica como tratamento: Revisão Bibliográfica. Rev Destaques Acadêmicos. 2019;11(3):93-9.

27. Dias L, Marçal L, Rodrigues M, Alves TCA, Pondé MP. Eficácia da Toxina Botulínica no Tratamento da Hiperidrose. Rev Neurociências. 2001;9(3):93-6.

28. Cohen PR. Scrotal Rejuvenation. Int Cureus. 2018;10(3):e2316.

29. Wu WTL. Microbotox of the lower face and neck: Evolution of a personal technique and its clinical effects. Int Plast Reconstr Surg. 2015;136(5):92S-100S.

30. Zhu J, Ji X, Xu Y, Liu J, Miao YY, Zhang JA, et al. The efficacy of intradermal injection of type $A$ botulinum toxin for facial rejuvenation. Int Dermatol Ther. 2017;30(1):10-3.

31. Oliveira GB, Rossi NCP, Moreira BMT. Tratamento da porção inferior do músculo orbicular dos olhos com microdoses de toxina botulínica: Série de 300 casos. Int Surg Cosmet Dermatology. 2016;8(3):206-9. 